\title{
Analysis of networks where discontinuities and nonsmooth dynamics collide: understanding synchrony
}

\author{
Yi Ming Lai, Rüdiger Thul, and Stephen Coombes ${ }^{\mathrm{a}}$ \\ Centre for Mathematical Medicine and Biology, School of Mathematical Sciences, \\ University of Nottingham, Nottingham, NG7 2RD, UK
}

Received 28 March 2018 / Received in final form 9 June 2018

Published online 12 December 2018

\begin{abstract}
Integrate-and-fire networks have proven remarkably useful in modelling the dynamics of real world phenomena ranging from earthquakes, to synchrony in neural networks, to cascading activity in social networks. The reset process means that such models are inherently discontinuous. Moreover, for jump interactions, which are a common choice for many physical systems, the models are also nonsmooth. For synchronous network states these processes can occur simultaneously, and care must be taken with the mathematical analysis of solution stability. This leads to an ordering problem, that has no counterpart in smoothly coupled limit cycle systems. Here we develop a set of network saltation matrices that can be used with an appropriate ordering to determine the instability of a synchronous network state. Moreover, we show that smoothed versions of jump interactions do not capture the behaviour of the nonsmooth model. Synchrony in the smoothed model with reset is analysed using a generalised master stability function $(\mathrm{MSF})$, and the eigenspectra for smooth and nonsmooth interactions are compared. We find that the one determined by the MSF organises that found from the analysis of the nonsmooth model, though the latter has further eigenvalues that can destabilise the synchronous state.
\end{abstract}

\section{Introduction}

The integrate-and-fire (IF) model has been a popular choice to describe an excitable or oscillatory neuron since the work of Bruce Knight in the 1960s [1]. Since then there have been several extensions of the model to allow for the generation of more physiologically realistic firing patterns, such as the model of Gröbler et al. [2] for a pyramidal cell in hippocampus CA3, and the Izhikevich model [3] which is now widely used throughout computational neuroscience for a wide variety of cell types. For a further discussion see [4]. At heart the IF model and its modern variants make use of a reset process to mimic the return to rest seen in a real neuron after the generation of an action potential. The time of reset is also used to signal the time that an action potential is generated. The IF model has also been invoked

\footnotetext{
a e-mail: stephen.coombes@nottingham.ac.uk
} 
outside of neuroscience, and typically to model systems with a threshold such as stickslip models of earthquakes [5], and social network models where a new behaviour is enacted by an individual once threshold is reached [6]. The act of reset means that IF models are inherently discontinuous. This is useful on one level since it means that an oscillator can be built from even a one dimensional dynamical system, rather than at least two dimensions as would be required for a smooth system in Euclidean space. On the other hand one cannot simply use the techniques developed for smooth dynamical systems, such as Floquet theory, to determine the stability of a periodic orbit. Fortunately, a set of techniques developed for the study of impact oscillators, such as those described by Müller to calculate Lyapunov exponents [7], allow for a natural extension of many of the techniques from smooth dynamical systems theory with the aid of saltation operators. In essence these describe how perturbations can be propagated through a discontinuity, allowing for a meaningful linear stability analysis of trajectories.

Networks built from IF nodes often make use of event times to generate interactions. For example, in a neural context a popular model of a first order synaptic current (which drives the IF dynamics) is one with a jump in a current followed by an exponential decay. Thus, in addition to reset, one has nonsmooth synaptic dynamics to contend with. Moreover, if an event time is defined by the time of reset then both processes can occur simultaneously, and discontinuities and nonsmooth dynamics can collide. Separation of the event and reset times by a delay can be used to circumvent this analytically challenging scenario. The focus of this paper is on developing an appropriate set of tools to handle the case that they occur simultaneously. In particular we are interested in the stability of synchronous states in networks of synaptically coupled IF neurons with arbitrary topology, subject to the constraint of a balance between excitation and inhibition. The case of purely pulsatile interactions has been considered elsewhere by many authors, e.g. [8-13], following the seminal work of Mirollo and Strogatz [14], and will not be considered further here.

In Section 2 we introduce the equations for our IF system and construct a synchronous orbit for a balanced network. We also introduce two forms of synaptic filter: (i) a first order filter for a nonsmooth synapse, and (ii) a second order filter for a smooth synapse. The latter has a limit that recovers the former and is useful for highlighting the similarities and differences between smooth and nonsmooth systems. An overview of linear stability for IF flows is given in Section 3, including how to generate the saltation operations to treat (linearised) flow through reset and across jump interactions. We first consider smooth synapses and show the master stability function (MSF) approach for smooth systems can be easily augmented to generate a spectral problem for the stability of the synchronous state. This spectrum is defined by the zeros of a complex function, and this function is succinctly expressed in terms of the IF node parameters and the Fourier transform of the synaptic filter. This provides a convenient backdrop with which to compare and contrast to the spectra for a network with nonsmooth synapses. Although there is no analogous MSF style approach for this case, here we show that an appropriate linear stability analysis can be developed by applying network saltation operations according to the order in which perturbed state variables at the node level cross threshold. Illustrative applications of the linear stability for both smooth and nonsmooth systems are presented in Section 4. Here we show that, although a smooth synaptic filter with a steep rise time might be thought to give qualitatively similar network behaviour as for one with a jump and decay, that this is not the case. In this limit we find that the spectra from the smooth analysis coincides with that from the nonsmooth one, yet the latter has further eigenvalues which can lead to different stability properties. Finally, in Section 5 we discuss natural extensions of the work presented in this paper. 


\section{IF networks with smooth and nonsmooth synaptic interactions}

The IF model has a long history of use in computational and mathematical neuroscience tracing its roots back to the work of Lapicque in 1907 [15]. Here we consider a network of IF nodes indexed by $i=1, \ldots, N$, each with a voltage variable $v_{i}=v_{i}(t) \leq v_{\mathrm{th}}, t \geq 0$. An IF neuron is reset such that just after the neuronal voltage reaches the threshold $v_{\text {th }}$ it is discontinuously changed to the reset value $v_{\mathrm{r}}<v_{\text {th }}$. Between reset and firing the voltage state variable of a (linear) IF neuron takes the simple form

$$
\frac{\mathrm{d}}{\mathrm{d} t} v_{i}=-\frac{v_{i}}{\tau_{m}}+I+I_{i}(t)
$$

for some constant drive $I$, and time-dependent input $I_{i}(t)$. The parameter $\tau_{m}$ is referred to as the single neuron membrane time constant (and note that for simplicity we only treat identical neurons). A set of firing times is defined according to the conditions

$$
T_{i}^{n}=\inf \left\{t \mid v_{i}(t) \geq v_{\text {th }} ; t \geq T_{i}^{n-1}\right\}, \quad n \in \mathbb{Z}
$$

The firing times are the cornerstone for describing event driven synaptic interactions. In this case the drive $I_{i}(t)$ represents the summed activity from other neural nodes in a network and is written

$$
I_{i}(t)=\sigma \sum_{j=1}^{N} W_{i j} s_{j}\left(t-\tau_{i j}\right),
$$

for some synaptic signal $s_{j}(t)$. Here $\sigma$ sets the overall scale of interaction strength, $W_{i j} \in \mathbb{R}$ specifies the specific strength of interaction between nodes $i$ and $j$, with a corresponding set of (axonal) communication delays $\tau_{i j} \in \mathbb{R}^{+}$. Motivated by the prevalence of balanced networks in neuroscience we restrict attention to the case that $\sum_{j=1}^{N} W_{i j}=0$ for all $i[16]$. The synaptic signal takes the explicit event driven form

$$
s_{j}(t)=\sum_{m \in \mathbb{Z}} \eta\left(t-T_{j}^{m}\right),
$$

where $\eta(t)$ is a normalised synaptic filter. This has the property that $\eta(t)=0$ for $t<0$ to ensure the causality of interactions. Many of the common choices for this filter that are employed in large scale computational neuroscience studies can be described using a Green's function formalism in the sense that there is a linear differential operator $Q$ such that $Q \eta(t)=\delta(t)$. For example, for the choice of an exponential synaptic response with $\eta(t)=\eta_{\alpha}(t)=\alpha \mathrm{e}^{-\alpha t} H(t)$, where $H$ is a Heaviside function, we have that $Q=Q_{\alpha}$ with

$$
Q_{\alpha}=\left(1+\frac{1}{\alpha} \frac{\mathrm{d}}{\mathrm{d} t}\right) .
$$

We shall refer to the exponential synapse model as nonsmooth since it is not continuous at $t=0\left(\eta_{\alpha} \notin C^{0}\right)$. In contrast we shall refer to the difference of exponentials function synapse with filter $\eta(t)=\eta_{\alpha \beta}(t)=\left(\alpha^{-1}-\beta^{-1}\right)^{-1}\left[\mathrm{e}^{-\alpha t}-\mathrm{e}^{-\beta t}\right] H(t)$ as smooth since it is continuous at $t=0\left(\eta_{\alpha \beta} \in C^{0}\right)$. The Green's function for this 
filter is easily calculated as $Q=Q_{\alpha \beta}=Q_{\alpha} Q_{\beta}$. The oft-encountered alpha function synapse is simply the case where $\alpha=\beta$.

For an overview of phase-locked states in IF networks and their analysis for smooth interactions we refer the reader to [17]. Here we focus on the analysis of the synchronous state. The balance condition ensures that a synchronous network state with $T_{i}^{m}=m \Delta$ for all $i$ can exist with $v_{i}(t)=v(t)$ where $v(t)$ is a $\Delta$-periodic solution, $v(t)=v(t+\Delta)$, that evolves according to

$$
\frac{\mathrm{d}}{\mathrm{d} t} v=-\frac{v}{\tau_{m}}+I, \quad 0 \leq t \leq \Delta .
$$

The period $\Delta$ is easily calculated by integrating the trajectory between reset and firing threshold, using $v(0)=v_{\mathrm{r}}$ and enforcing the constraint $v(\Delta)=v_{\text {th }}$ to yield $\Delta=\tau_{m} \log \left[\left(I \tau_{m}-v_{\mathrm{r}}\right) /\left(I \tau_{m}-v_{\mathrm{th}}\right)\right] H\left(I \tau_{m}-v_{\mathrm{th}}\right)$. To determine the linear stability of the synchronous state one must consider the evolution of small perturbations through the firing and reset process and their effects on event driven interactions. We shall now exploit the sub-threshold linearity of the model to show how the full nonsmooth flow of the network can be constructed by augmenting the smooth sub-threshold flow with a set of appropriate saltation operations.

\section{From smooth to nonsmooth analysis}

Saltation matrices have been used extensively in the study of nonsmooth mechanical systems, and for an overview see [18]. Their use for studying IF dynamics is less common, though they have previously been used to construct Liapunov exponents to probe chaotic response $[19,20]$. We begin with a discussion that highlights their need, and derive the form of the saltation rules that can correctly map linearised flows through events (reset or interaction). We then consider smooth synapses for which we need only treat saltation through reset and construct the MSF. For nonsmooth synapses a quite different approach is needed to determine the stability of the synchronous state that takes into account the possibility that perturbed node trajectories can overtake one another.

\subsection{Saltation operators for IF networks}

The effect of reset can be incorporated into the dynamical description given by (1) under the replacement $I_{i}(t) \rightarrow I_{i}(t)-\left(v_{\mathrm{th}}-v_{\mathrm{r}}\right) \sum_{m \leq M(i, t)} \delta\left(t-T_{i}^{m}\right)$, where $M(i, t)$ indexes the last time that node $i$ fired before time $t$. A variation of parameters method can then be used to integrate the dynamics up to time $t$ as

$$
\begin{aligned}
v_{i}(t)= & \mathrm{e}^{-t / \tau_{m}} v_{i}(0)+I \tau_{m}\left(1-\mathrm{e}^{-t / \tau_{m}}\right)+\sigma \sum_{j=1}^{N} W_{i j} \sum_{m \leq M(j, t)} \psi\left(t-T_{j}^{m}-\tau_{i j}\right) \\
& -\left(v_{\mathrm{th}}-v_{\mathrm{r}}\right) \sum_{m \leq M(i, t)} \mathrm{e}^{-\left(t-T_{i}^{m}\right) / \tau_{m}} .
\end{aligned}
$$

Here $\psi$ is a new temporal filter given by the convolution:

$$
\psi(t)=\int_{0}^{t} \mathrm{e}^{-(t-s) / \tau_{m}} \eta(s) \mathrm{d} s .
$$


We may now treat small perturbations around an arbitrary trajectory $v_{i}(t)$ by considering $\widetilde{v}_{i}(0)=v_{i}(0)+\delta v_{i}(0)$ and $T_{i}^{m} \rightarrow T_{i}^{m}+\delta T_{i}^{m}$, with $\delta T_{i}^{m} \ll 1$. Hence, using (7), we have to first order in $\delta T_{i}^{m}$ that

$$
\begin{aligned}
\delta v_{i}(t)= & \delta v_{i}(0) \mathrm{e}^{-t / \tau_{m}}-\sigma \sum_{j=1}^{N} W_{i j} \sum_{m \leq M(j, t)} \psi^{\prime}\left(t-T_{j}^{m}-\tau_{i j}\right) \delta T_{j}^{m} \\
& -\frac{\left(v_{\mathrm{th}}-v_{\mathrm{r}}\right)}{\tau_{m}} \sum_{m \leq M(i, t)} \mathrm{e}^{-\left(t-T_{i}^{m}\right) / \tau_{m}} \delta T_{i}^{m}
\end{aligned}
$$

where $\delta v_{i}(t)=\widetilde{v}_{i}(t)-v_{i}(t)$. It is a simple matter to show that $\psi^{\prime}(t)=\eta(t)-\psi(t) / \tau_{m}$. The perturbations of the threshold crossings may be calculated by Taylor expansion of the threshold condition $\widetilde{v}_{i}\left(T_{i}^{m}+\delta T_{i}^{m}\right)=v_{\text {th }}$. Denoting the difference between the perturbed and unperturbed trajectories just before the firing event at $T_{j}^{m}$ as $\delta v_{i}^{-}\left(T_{j}^{m}\right)$ and that after as $\delta v_{i}^{+}\left(T_{j}^{m}\right)$ we have to first order that

$$
\delta T_{i}^{m}=-\frac{\delta v_{i}^{-}\left(T_{i}^{m}\right)}{\widetilde{v}_{i}^{\prime}\left(T_{i}^{m-}\right)}
$$

Hence, firing events in the network cause the perturbations $\delta v_{i}(t)$ to jump discontinuously to some new value according to equations (9) and (10). We find that the jump induced by reset is given by

$$
\delta v_{i}^{+}\left(T_{i}^{m}\right)=\left[1+\frac{\left(v_{\mathrm{th}}-v_{\mathrm{r}}\right) / \tau_{m}}{\widetilde{v}_{i}^{\prime}\left(T_{i}^{m-}\right)}\right] \delta v_{i}^{-}\left(T_{i}^{m}\right)
$$

and the jump induced by interaction is given by

$$
\delta v_{i}^{+}\left(T_{j}^{m}+\tau_{i j}\right)=\delta v_{i}^{-}\left(T_{j}^{m}+\tau_{i j}\right)+\sigma W_{i j} \frac{\psi^{\prime}(0)}{\widetilde{v}_{j}^{\prime}\left(T_{j}^{m-}\right)} \delta v_{j}\left(T_{j}^{m}\right)
$$

Equations (11) and (12) provide the rules for saltation, and to close the equations we see that we need rules for estimating $\widetilde{v}_{i}^{\prime}$. For systems with smooth synaptic interactions the natural estimate is $\widetilde{v}_{i}^{\prime} \simeq v_{i}^{\prime}$, though for nonsmooth interactions one needs to be more careful. Moreover, from (12) we see that if $\psi^{\prime}(0)=0$ then there are no jumps due to synaptic interactions, and we only have to consider those from reset. For an exponential synapse $\psi^{\prime}(0)=\eta_{\alpha}(0)=\alpha$, whilst for a difference of exponentials function $\psi^{\prime}(0)=\eta_{\alpha \beta}(0)=0$. In the former case we must be very careful when considering issues of linear stability for zero delays $\left(\tau_{i j}=0\right.$ for all $\left.i, j\right)$ because there are two types of jump that can occur simultaneously in time. For the latter case one need only treat jumps arising from reset. This suggests that the stability analysis of network states for nonsmooth synapses (such as an exponential function) cannot be treated with the same tools as for smooth synapses (such as a difference of exponentials function), at least for the case of zero delays. We now present two different approaches for assessing the stability of the synchronous state, one approach for smooth synapses and the other for nonsmooth. 


\subsection{MSF for smooth synapses}

In essence the MSF is a tool for assessing the linear stability of synchronous states in networks of smoothly coupled oscillators (typically with a graph Laplacian form of coupling) [21]. It is highly practical since the network stability is determined in terms of a reduced system, with a dimension the same as that of the phase-space for a single node, parametrised by an eigenvalue of the coupling matrix. This means that once the MSF is constructed it can be used to assess the stability of the synchronous state in a network of arbitrary topology. In previous work Ladenbauer et al. [22] have developed an MSF method to classify synchronous states of a spiking neuronal network with delayed nonsmooth interactions (and the delay ensures that the reset and jumps do not collide). They focused on a nonlinear planar IF node, for which periodic orbits and Floquet multipliers needed to be computed numerically. The linear IF model considered here is much simpler and far more analytical progress can be made.

For simplicity we shall focus on the choice of a common delay and set $\tau_{i j}=\tau \geq 0$ for all $i, j$, and absorb this single delay into an effective synaptic filter $\zeta(t)=\eta(t-\tau)$. We consider the synchronous state as discussed in Section 2, with $s_{i}(t)=s(t)$ for all $i$ and $s_{i}(t)=\sum_{m \in \mathbb{Z}} \zeta(t-m \Delta)$. The variational equation around the synchronous solution, (obtained by considering $\left(v_{i}(t), s_{i}(t)\right)=(v(t), s(t))+\left(\delta v_{i}(t), \delta s_{i}(t)\right)$, and $\left.T_{i}^{m}=m \Delta+\delta T_{i}^{m}\right)$, takes the form

$$
\frac{\mathrm{d}}{\mathrm{d} t} \delta v_{i}=-\frac{\delta v_{i}}{\tau_{m}}+\sigma \sum_{j=1}^{N} W_{i j} \delta s_{j}
$$

where

$$
\delta s_{i}(t)=\sum_{m \in \mathbb{Z}} \zeta^{\prime}(t+m \Delta)\left(-\delta T_{i}^{-m}\right)
$$

From (10) (and using the synchronous orbit as a reference trajectory with $v_{i}^{\prime}=v^{\prime}$ ) we have that $\delta T_{i}^{m}=-\delta v_{i}^{m} / v^{\prime}\left(\Delta^{-}\right)$, where $\delta v_{i}^{m}=\delta v_{i}^{-}(m \Delta)$. Thus, we have a history dependent variational problem that can be solved with the choice

$$
\delta v_{i}^{m}=\delta v_{i}(0) \lambda^{m}, \quad \lambda \in \mathbb{C} .
$$

A computationally useful form of (14) can be obtained using an integral representation for $\zeta(t)$ as

$$
\zeta(t)=\frac{1}{2 \pi} \int_{-\infty}^{\infty} \mathrm{d} \omega \mathrm{e}^{i \omega t} \widetilde{\zeta}(\omega)
$$

In this case we have that

$$
\delta s_{i}(t)=\frac{\delta v_{i}(0)}{v^{\prime}\left(\Delta^{-}\right)} \sum_{m \in \mathbb{Z}} \lambda^{-m} \int_{-\infty}^{\infty} \frac{\mathrm{d} \omega}{2 \pi} \mathrm{e}^{i \omega(t+m \Delta)} i \omega \widetilde{\zeta}(\omega) .
$$

To evaluate the term $\sum_{m \in \mathbb{Z}} \lambda^{-m} \mathrm{e}^{i m \Delta}$ we use the formal (Dirac-comb) result that

$$
\sum_{m \in \mathbb{Z}} \mathrm{e}^{i x m \Delta}=\frac{2 \pi}{\Delta} \sum_{n \in \mathbb{Z}} \delta(x-2 \pi n / \Delta),
$$


and set $x=i \Delta^{-1} \ln \lambda+\omega$ in (17) to obtain

$$
\delta s_{i}(t)=\frac{\delta v_{i}(0)}{v^{\prime}\left(\Delta^{-}\right)} \frac{1}{\Delta} \sum_{n \in \mathbb{Z}} \widetilde{\zeta}\left(\omega_{n}-i \gamma\right)\left(\gamma+i \omega_{n}\right) \mathrm{e}^{\left(\gamma+i \omega_{n}\right) t}
$$

Here we have introduced the Floquet exponent $\gamma=\Delta^{-1} \ln \lambda$ and $\omega_{n}=2 \pi n / \Delta$. If we define a vector $\delta v=\left(\delta v_{1}, \ldots, \delta v_{N}\right)$ and a matrix that is the eigenvectors of the matrix $W$ (with components $W_{i j}$ ), with $W P=P \Lambda$, where $\Lambda=\operatorname{diag}\left(\nu_{1}, \ldots, \nu_{N}\right)$ is the associated diagonal matrix of eigenvalues, then (13) can be diagonalised to the form

$$
\frac{\mathrm{d}}{\mathrm{d} t} \delta u(t)=-\frac{\delta u(t)}{\tau_{m}}+\sigma \Lambda \frac{F(t, \gamma)}{v^{\prime}\left(\Delta^{-}\right)} \delta u(0)
$$

where $\delta u=P^{-1} \delta v$, and $F(t, \gamma)=\Delta^{-1} \sum_{n} \widetilde{\zeta}\left(\omega_{n}-i \gamma\right)\left(\gamma+i \omega_{n}\right) \mathrm{e}^{\left(\gamma+i \omega_{n}\right) t}$. We may integrate the above between firing to give

$$
\delta u_{i}(\Delta)=\delta u_{i}\left(0^{+}\right) \mathrm{e}^{-\Delta / \tau_{m}}+\sigma \frac{\nu_{i}}{v^{\prime}\left(\Delta^{-}\right)} G(\gamma) \delta u_{i}(0)
$$

where

$$
G(\gamma)=\mathrm{e}^{-\Delta / \tau_{m}} \int_{0}^{\Delta} \mathrm{d} s \mathrm{e}^{s / \tau_{m}} F(s, \gamma)=\frac{1}{\Delta} \sum_{n \in \mathbb{Z}} \frac{\widetilde{\zeta}\left(\omega_{n}-i \gamma\right)\left(\gamma+i \omega_{n}\right)\left(\mathrm{e}^{\gamma \Delta}-\mathrm{e}^{-\Delta / \tau_{m}}\right)}{\tau_{m}^{-1}+\gamma+i \omega_{n}}
$$

Now we can apply the saltation operator (11) to construct $\delta u_{i}\left(0^{+}\right)=\kappa \delta u_{i}(0)$, where $\kappa=v^{\prime}\left(\Delta^{+}\right) / v^{\prime}\left(\Delta^{-}\right)$. Rearranging, and remembering $\delta u_{i}(\Delta)=\lambda \delta u_{i}(0)$, then gives

$$
\left\{(\lambda-1) v^{\prime}\left(\Delta^{-}\right)-\sigma \nu_{i} G(\ln \lambda / \Delta)\right\} \delta u_{i}(0)=0,
$$

where we have used the result that $v^{\prime}\left(\Delta^{+}\right) / v^{\prime}\left(\Delta^{-}\right) \mathrm{e}^{-\Delta / \tau_{m}}=1$. Hence the MSF can be defined in terms of the roots of $\mathcal{E}(\lambda ; \chi)=0$, where $\chi \in \mathbb{C}$ and

$$
\mathcal{E}(\lambda ; \chi)=(\lambda-1)\left(-v_{\text {th }} / \tau_{m}+I\right)-\chi G(\ln \lambda / \Delta) .
$$

We see that there is a root with $\lambda=1$ for $\chi=0$ as expected. The MSF is the largest real part of the Floquet exponent $\gamma=\Delta^{-1} \ln \lambda$, and the synchronous state is stable if the MSF is negative at all the points where $\chi=\sigma \nu_{i}$. We note that this recovers a previous result in [17] obtained using a firing-map argument. Thus to determine the stability of the synchronous state we need only to prescribe the Fourier transform of the synaptic filter. For the delayed exponential synapse $\eta(t)=\eta_{\alpha}(t)$ we have that $\widetilde{\zeta}(\omega)=\alpha \mathrm{e}^{-i \omega \tau} /(\alpha+i \omega)$, and for the difference of exponentials choice $\eta(t)=\eta_{\alpha \beta}(t)$ we have that $\widetilde{\zeta}(\omega)=\alpha \beta \mathrm{e}^{-i \omega \tau} /[(\alpha+i \omega)(\beta+i \omega)]$.

In Figure 1 we show plots of the MSF for the case of zero and non-zero delays. We observe that the difference of exponentials synapse (a) with $\alpha=1$ and $\beta=10$ is qualitatively similar to the alpha synapse with $\alpha=1$ (c). The MSF for the difference of exponentials synapse (a) is also almost identical to the MSF for the delayed exponential synapse (b) with delay $\tau=0.1$. This is in line with our intuition, as for $\beta \gg \alpha$, the difference of exponentials synapse has a sharp rise with a rise time of approximately $\beta^{-1}$, before decaying exponentially with a rate close to $\alpha$. For larger delays, the MSF can change shape quite substantially as shown in (d) for a delayed exponential synapse with $\tau=0.5$. 
(a)

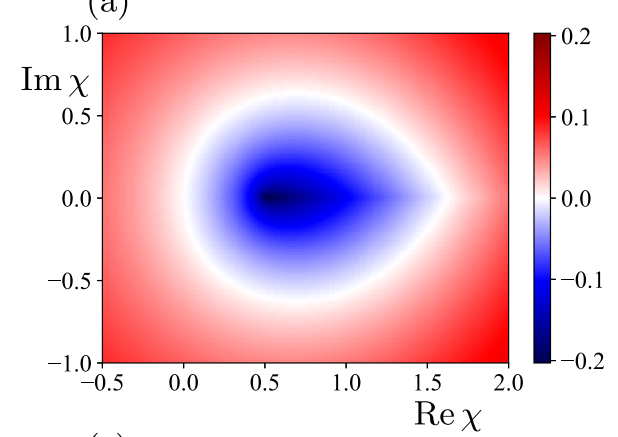

(c)

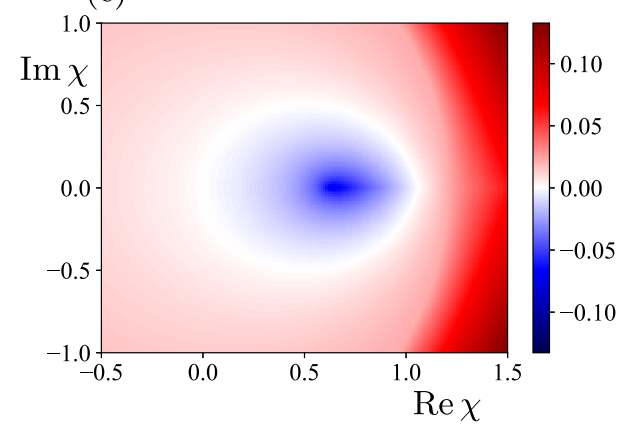

(b)

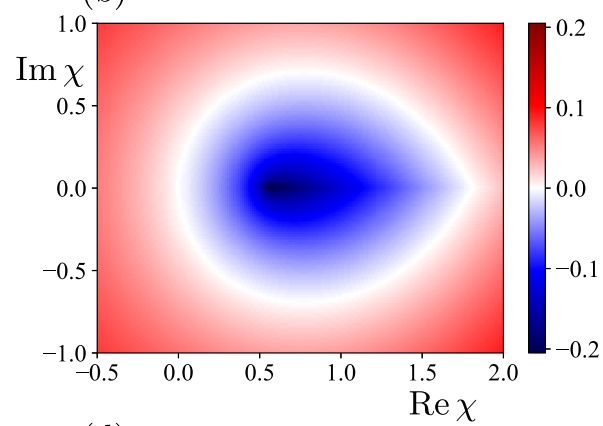

(d)

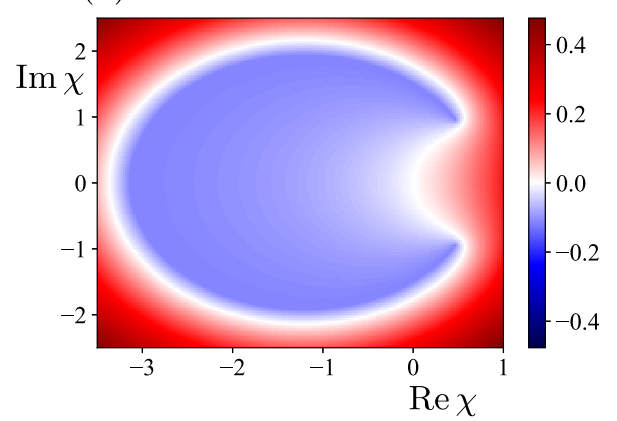

Fig. 1. MSF for different synaptic filters. The colours correspond to the MSF, so that negative (blue) regions indicate stability. (a) Difference of exponentials synapse with $(\alpha, \beta)=$ $(1,10)$. (b) Delayed exponential synapse with $(\alpha, \tau)=(1,0.1)$. (c) Alpha synapse with $\alpha=\beta$ and $\alpha=1$. (d) Delayed exponential synapse with $(\alpha, \tau)=(1,0.5)$. Other parameters: $v_{\mathrm{r}}=0$, $v_{\text {th }}=1, I=2$, and $\tau_{m}=1$.

\subsection{Ordering for nonsmooth synapses}

In pulse-coupled networks trajectories of nodes may jump over one another giving rise to an ordering problem when considering linear stability [13,23]. The same issue occurs for non-pulsatile forcing where nonsmooth interactions can cause jumps in the velocities of network trajectories, and one must be careful when propagating perturbations around a synchronous solution. If one component of a perturbed network state crosses threshold it will affect the gradients of the other components. To develop a computationally useful framework for using saltation operations in a setting with velocity jumps it is more natural to consider the network evolution as a whole, rather than attempt to diagonalise it as done in Section 3.2. This has recently been done for a network of piecewise linear neural mass models [24], and here we adapt this approach to treat the spiking nature of IF processes. For clarity of exposition we shall focus on the case of an exponential synapse and no delays.

We introduce a vector $z=\left(v_{1}, \ldots, v_{N}, s_{1}, \ldots, s_{N}\right) \in \mathbb{R}^{2 N}$ and write the synchronous state as $\bar{z}=(v, \ldots, v, s, \ldots, s)$ and a perturbed state as $\widetilde{z}=$ $\left(\widetilde{v}_{1}, \ldots, \widetilde{v}_{N}, \widetilde{s}_{1}, \ldots, \widetilde{s}_{N}\right)$. For an exponential synapse $s(t)=s_{0} \mathrm{e}^{-\alpha t}$ for $0 \leq t<\Delta$ with $s(t)=s(t+\Delta)$ and $s_{0}=\alpha /\left(1-\mathrm{e}^{-\alpha \Delta}\right)$. We define a set of perturbed firing times according to $\widetilde{T}_{i}^{m}=T_{i}^{m}+\delta T_{i}^{m}$, where $T_{i}^{m}=m \Delta$ for all $i$ and $\delta T_{i}^{m} \ll 1$. We shall now consider an ordering of the perturbed firing times around the time $m \Delta$ such that $\delta T_{p_{1}}^{m}<\delta T_{p_{2}}^{m}<\ldots<\delta T_{p_{N}}^{m}<0$. Here $p$ is a permutation of the node indices such that $p_{1}$ represents the index of the component of the perturbed network trajectory that reaches threshold first, and $p_{N}$ the component that reaches threshold last. For 


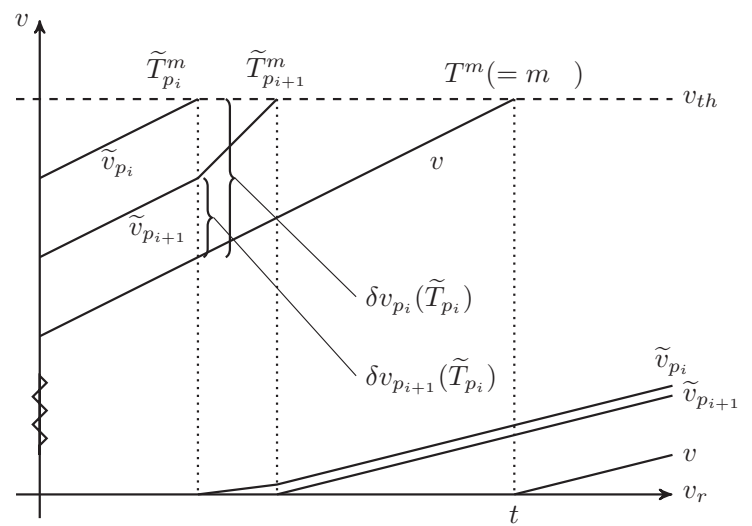

Fig. 2. Schematic showing how the voltage gradient of trajectories evolves through the threshold process, and a depiction of the various perturbed voltage components and perturbed event times used in the linear stability analysis.

$\widetilde{T}_{p_{i}}^{m} \leq t<\widetilde{T}_{p_{i+1}}^{m}$ the perturbed system is continuous and we have that

$$
\delta z\left(\widetilde{T}_{p_{i+1}}^{m}\right)=\delta z\left(\widetilde{T}_{p_{i}}^{m}\right)+\left[\widetilde{z}^{\prime}\left(\widetilde{T}_{p_{i}}^{m}\right)-\bar{z}^{\prime}\left(\widetilde{T}_{p_{i}}^{m}\right)\right]\left(\delta T_{p_{i+1}}^{m}-\delta T_{p_{i}}^{m}\right),
$$

where $\delta z=\widetilde{z}-\bar{z}$. In Figure 2 we provide a schematic showing how components of the perturbed network trajectory are affected by reset. Initially all voltage components have the same velocity as the synchronous orbit. At time $\widetilde{T}_{p_{i}}^{m}$ neuron $p_{i}$ reaches threshold and resets. Due to nonsmooth interactions neuron $p_{i+1}$ experiences an instantaneous change in its voltage gradient. After all neurons have crossed threshold they all have the same velocity as the synchronous orbit (to first order) due to the balanced nature of interactions. The time difference $\delta T_{p_{i+1}}^{m}-\delta T_{p_{i}}^{m}$ between events can be computed by considering the equation $v\left(\widetilde{T}_{p_{i}}\right)=v_{\mathrm{th}}-\delta v_{p_{i}}\left(\widetilde{T}_{p_{i}}\right)=\widetilde{v}_{p_{i+1}}\left(\widetilde{T}_{p_{i}}\right)-$ $\delta v_{p_{i+1}}\left(\widetilde{T}_{p_{i}}\right)$ and Taylor expanding around $\widetilde{T}_{p_{i+1}}$ to give

$$
\delta T_{p_{i}}^{m}-\delta T_{p_{i+1}}^{m}=\frac{\delta v_{p_{i+1}}\left(\widetilde{T}_{p_{i}}^{m}\right)-\delta v_{p_{i}}\left(\widetilde{T}_{p_{i}}^{m}\right)}{\widetilde{v}_{p_{i+1}}^{\prime}\left(\widetilde{T}_{p_{i+1}}\right)}
$$

where we set $\delta T_{p_{N+1}}=0=\delta v_{p_{N+1}}$. The natural estimator of $\widetilde{v}_{p_{j}}^{\prime}\left(\widetilde{T}_{p_{i+1}}^{m}\right)$ is

$$
\widetilde{v}_{p_{j}}^{\prime}\left(\widetilde{T}_{p_{i+1}}^{m}\right)= \begin{cases}\widetilde{v}_{p_{j}}^{\prime}\left(\widetilde{T}_{p_{i}}^{m}\right)+\alpha \sigma W_{p_{j}, p_{i}} & p_{j} \geq p_{i+1} \\ -v_{\mathrm{r}} / \tau_{m}+I+\alpha \sigma\left[W_{p_{j}, p_{j}}+W_{p_{j}, p_{i}}\right] & p_{j}<p_{i+1}\end{cases}
$$

Here $\widetilde{v}_{i}^{\prime}\left(\widetilde{T}_{p_{1}}^{m}\right) \simeq \bar{v}^{\prime}\left(\Delta^{-}\right)=-v_{\text {th }} / \tau_{m}+I$ for all $i=1, \ldots, N$.

Similarly, the natural estimator of $\widetilde{s}_{j}^{\prime}\left(\widetilde{T}_{p_{i+1}}^{m}\right)$ (for an exponential synapse) is $\widetilde{s}_{j}^{\prime}\left(\widetilde{T}_{p_{i+1}}^{m}\right)=\widetilde{s}_{j}^{\prime}\left(\widetilde{T}_{p_{i}}^{m}\right)$, for $j \neq p_{i}$, and

$$
\widetilde{s}_{p_{i}}^{\prime}\left(\widetilde{T}_{p_{i+1}}^{m}\right)=\widetilde{s}_{p_{i}}^{\prime}\left(\widetilde{T}_{p_{i}}^{m}\right)-\alpha^{2} .
$$

Here, $\widetilde{s}_{i}^{\prime}\left(\widetilde{T}_{p_{1}}^{m}\right) \simeq \bar{s}^{\prime}\left(\Delta^{-}\right)=-\alpha s_{0} \mathrm{e}^{-\alpha \Delta}$ for all $i=1, \ldots, N$. Equations (27) and (28) capture the jump dependence of the perturbed system, and also allow us to construct 
the vector $\widetilde{z}^{\prime}\left(\widetilde{T}_{p_{i}}^{m}\right)$ that appears in $(25)$. Using the above we may now write $(25)$ in the succinct form

$$
\delta z\left(\widetilde{T}_{p_{i+1}}^{m}\right)=K_{m}\left(p_{i}\right) \delta z\left(\widetilde{T}_{p_{i}}^{m}\right), \quad i=1, \ldots, N
$$

where we set $\widetilde{T}_{p_{N+1}}^{m}=m \Delta$ and $K_{m}\left(p_{i}\right)$ is the network saltation matrix:

$$
K_{m}\left(p_{i}\right)= \begin{cases}I_{2 N}-\frac{\left(\widetilde{z}^{\prime}\left(\widetilde{T}_{p_{i}}^{m}\right)-\bar{z}^{\prime}\left(\widetilde{T}_{p_{i}}^{m}\right)\right) e_{p_{i}}^{\top}}{\bar{v}^{\prime}\left(\Delta^{-}\right)} & i=N \\ I_{2 N}+\frac{\left(\widetilde{z}^{\prime}\left(\widetilde{T}_{p_{i}}^{m}\right)-\bar{z}^{\prime}\left(\widetilde{T}_{p_{i}}^{m}\right)\left(e_{p_{i}}^{\top}-e_{p_{i+1}}^{\top}\right)\right.}{\bar{v}^{\prime}\left(\Delta^{-}\right)+\alpha \sigma \sum_{j=1}^{i} W_{p_{j+1}, p_{j}}} & i \neq N .\end{cases}
$$

Here $e_{m}=\left(f_{m}, O\right) \in \mathbb{R}^{2 N}$ with $f_{m}$ a canonical basis vector in $\mathbb{R}^{N}$ and $O$ the zero vector in $\mathbb{R}^{N}$. For times away from firing the linearised dynamics takes the simple form

$$
\frac{\mathrm{d}}{\mathrm{d} t} \delta z=J \delta z
$$

where the Jacobian $J \in \mathbb{R}^{2 N \times 2 N}$ has a block structure:

$$
J=\left[\begin{array}{cc}
-I_{N} / \tau & \sigma W \\
0_{N} & -\alpha I_{N}
\end{array}\right]
$$

and $0_{N}$ denotes the $N \times N$ zero matrix. Thus, we may evolve an initial network perturbation $\delta z(0)$ up to some time $m \Delta$ using $\delta z(m \Delta)=\Gamma(m) \delta z(0)$, with

$$
\begin{aligned}
\Gamma(m)= & K_{m}\left(p_{N}\right) \cdots K_{m}\left(p_{1}\right) G(\Delta) K_{m-1}\left(p_{N}\right) \cdots K_{m-1}\left(p_{1}\right) \\
& \times G(\Delta) \cdots K_{2}\left(p_{N}\right) \cdots K_{2}\left(p_{1}\right) G(\Delta) K_{1}\left(p_{N}\right) \cdots K_{1}\left(p_{1}\right) G(\Delta),
\end{aligned}
$$

where $G(t)=\exp (J t)$. If the order of firing is fixed for all $m$ then $K_{m}\left(p_{i}\right)=K_{1}\left(p_{i}\right)$ for all $m$ and $\Gamma(m)=\kappa^{m}, \kappa=K_{1}\left(p_{N}\right) \ldots K_{1}\left(p_{1}\right) G(\Delta)$, and stability can be determined in terms of the eigenvalues of the matrix $\kappa$, with synchrony stable if all the eigenvalues of $\kappa$ (excluding the one of unity arising from time-translation symmetry) lie within the unit disc in the complex plane. However, a persistent ordering of firing times from cycle to cycle is non-generic and instead must be determined self-consistently. This means that there is no simple prescription for determining stability. In a practical sense one can consider the spectrum of $\Gamma(m)$ for large $m$ and consider synchrony to be stable if all eigenvalues lie within the unit disc for a large set of initial random perturbations.

\section{Examples}

To help contrast the differing network behaviours arising from nonsmooth and smooth synaptic filters we consider the limit $\beta \rightarrow \infty$ for a difference of exponentials synaptic filter. In this case the nonsmooth exponential filter is recovered from the smooth difference of exponentials filter:

$$
\eta_{\alpha}(t)=\lim _{\beta \rightarrow \infty} \eta_{\alpha \beta}(t)
$$


(a)

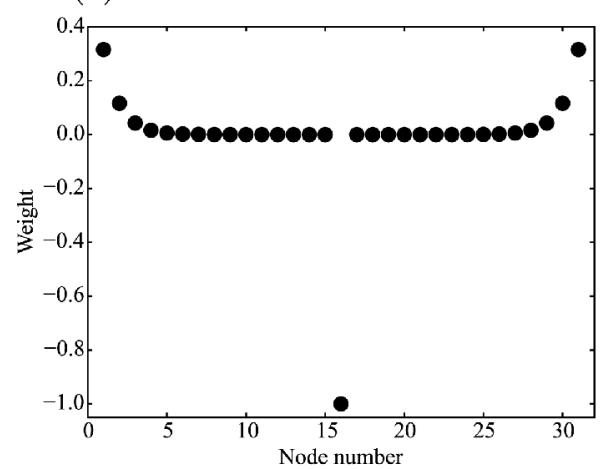

(c)

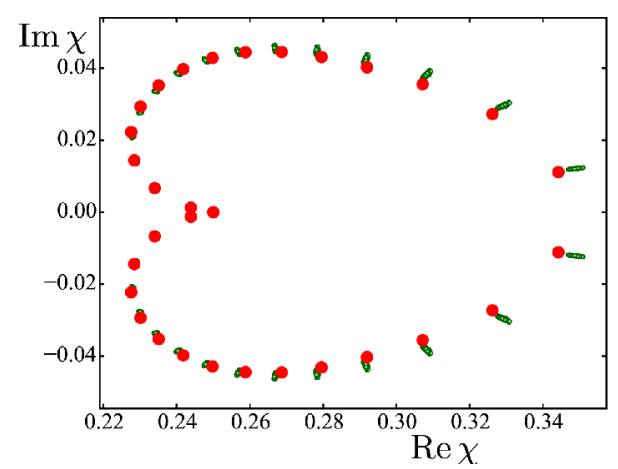

(b)

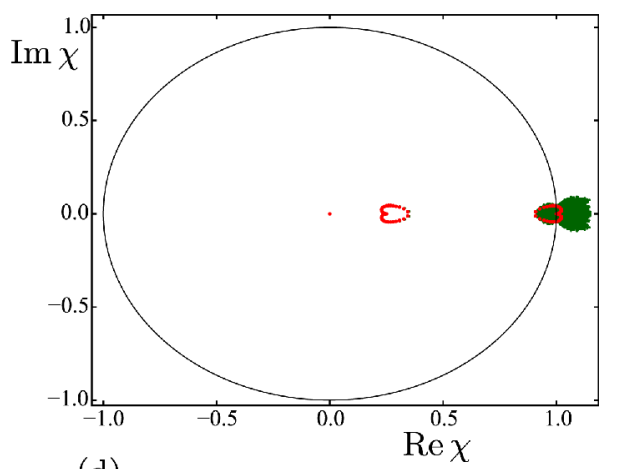

(d)

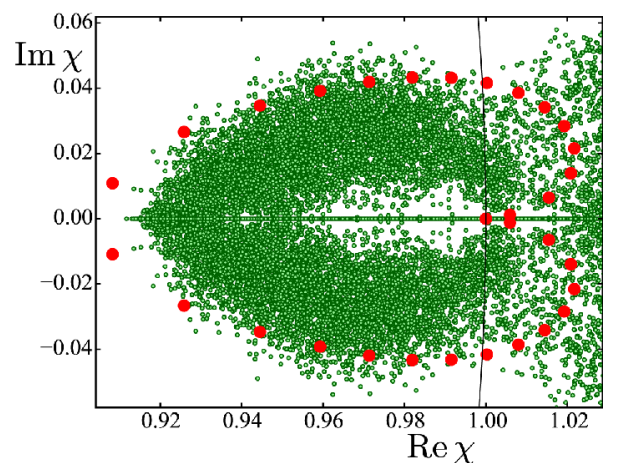

Fig. 3. Spectral analysis of the synchronous state in an IF ring network with nonsmooth synapses for $N=31$ nodes. (a) Graph showing the functional form of a single row in a balanced circulant matrix using (36) with $d=1$. (b) The eigenspectrum for the case of a nonsmooth exponential synapse with $\alpha=2$ is shown with green circles. The eigenspectrum for a smooth difference of exponentials synapse with $(\alpha, \beta)=(2,100)$ is shown with red circles. (c) A zoom of (b) interior to the unit disc. (d) A zoom of (b) on the edge of the unit disc. The zooms show that the eigenvalues of the nonsmooth system cluster around those of the smooth system. Other parameters as in Figure 1 with $\sigma=0.125$.

We first consider a ring with an odd number of nodes and a strength of interactions that depends on the distance between nodes as illustrated in Figure 3a. The strength of interactions is given by $W_{i j}=w_{(i+j-1)} \bmod N$, where the $w_{i}$ are chosen such that

$$
\begin{aligned}
\left\{w_{1}, w_{2}, \ldots, w_{(N-1) / 2}\right\} & =\left\{w_{N}, w_{N-1}, \ldots, w_{N+1-(N-1) / 2}\right\} \\
& =S^{-1}\left\{\mathrm{e}^{-d}, \mathrm{e}^{-2 d}, \ldots, \mathrm{e}^{-d(N-1) / 2}\right\}
\end{aligned}
$$

which is balanced by the choice of $w_{(N-1) / 2+1}=-1$ and a constant $S=$ $\sum_{k=1}^{(N-1) / 2} \mathrm{e}^{-d k}$. We find that the eigenvalues of the nonsmooth (exponential synapse) system cluster around those of the smooth system (difference of exponentials with large $\beta$ ). The nonsmooth system has (combinatorially) more eigenvalues than those from the smooth system and they can reside outside of the unit circle. This reaffirms the notion that it can be dangerous to use a smoothed system to predict the behaviour of a truly nonsmooth system. Changing the scaling $d$ has no qualitative effect on the stability of the synchronous state. 
(a)

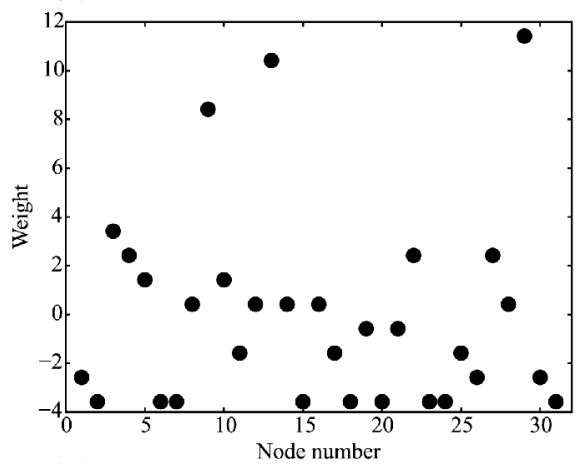

(c)

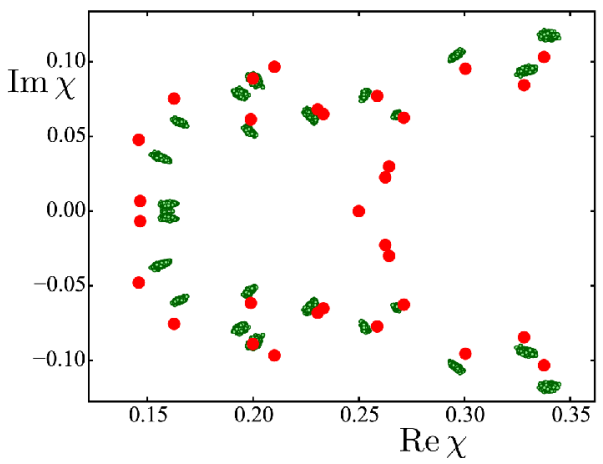

(b)

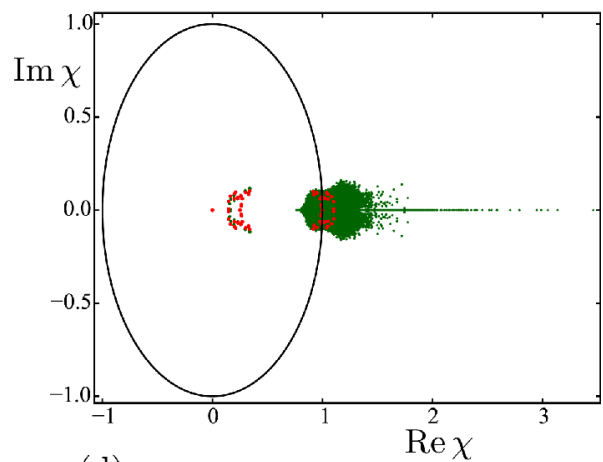

(d)

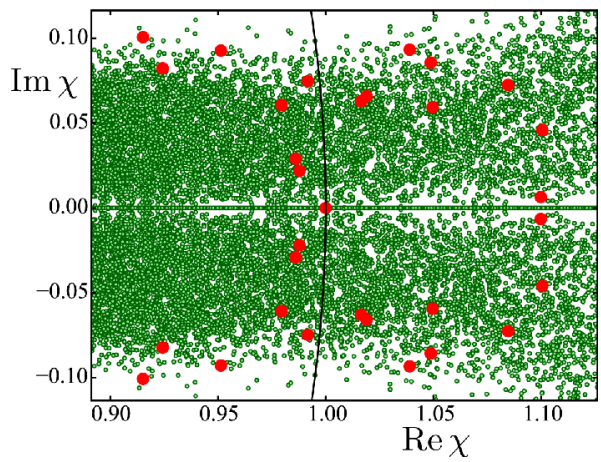

Fig. 4. Spectral analysis of the synchronous state in an IF ring network with nonsmooth synapses for $N=31$ nodes. (a) Graph showing the functional form of a single row in a balanced circulant matrix with components $w_{i}, i=1, \ldots, N$, such that $W_{i j}=w_{(i+j-1) \bmod N}$. Here the $w_{i}$ are chosen such that $\operatorname{Pr}(w=k)=(1-p)^{k-1} p$ with $p=0.2$ (a geometric distribution), and then shifted to ensure a balanced network. (b) The eigenspectrum for the case of an exponential synapse with $\alpha=2$ is shown with green circles. The eigenspectrum from a smooth difference of exponentials synapse with $(\alpha, \beta)=(2,100)$ is shown with red circles. (c) A zoom of (b) interior to the unit disc. (d) A zoom of (b) on the edge of the unit disc. The zooms show that the eigenvalues of the nonsmooth system cluster around those of the smooth system. Other parameters as in Figure 1 with $\sigma=0.0125$.

We next consider a ring with randomly generated weights. We take $N$ i.i.d variables from a geometric distribution, then subtract the arithmetic mean to obtain a balanced interaction row vector (Fig. 4a). We then generate a circulant coupling matrix using this weight vector. The spectra from the linear stability analysis is shown in Figure 4. Here we also find that the spectra from the nonsmooth system is organised around that from a smooth system. The former is obtained by considering 1000 randomly generated threshold crossings. We see that inside the unit disc, eigenvalues of the smooth system tend to cluster around those of the nonsmooth system, but their structure is much less organised outside the unit disc.

Finally, we consider a globally coupled network with nonsmooth synapses and $\sigma>0$. Similarly to the case of pulse-coupled oscillators [12], we find that the stability of the synchronous state can change with the number of neurons in the network. In contrast, for smooth synapses the stability is determined entirely by the eigenvalues of the graph Laplacian, which do not vary as a function of $N$ (for a globally coupled network). For this special case (with permutation symmetry) all possible orderings 


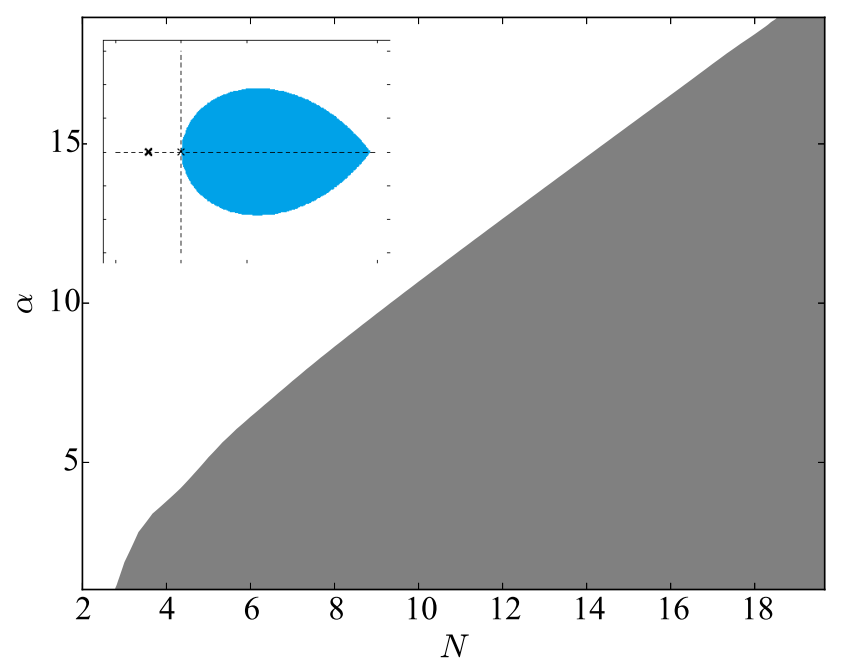

Fig. 5. A plot showing the stability of the synchronous state in a globally coupled balanced network with $W_{i j}=1 / N-\delta_{i j}$ and an exponential synapse in the $(\alpha, N)$ plane according to the nonsmooth linear stability analysis. The grey shaded region indicates unstable and the white stable. The inset shows a plot of the MSF (only negative where coloured blue) for a smooth difference of exponentials synapse with a fast rise time, namely with $(\alpha, \beta)=(2,100)$. The cross indicates the $(N-1$ degenerate) eigenvalue at -1 for the graph Laplacian of the globally coupled network. Parameters as in Figure 1 with $\sigma=0.2$.

lead to the same stability calculation. We exploit the fact that we need only compute one set of eigenvalues and sweep the parameter space of $\alpha$ and $N$ to determine stability, and show the results in Figure 5. We discover that for a given $\alpha$, networks below a critical size have a stable synchronous state, and that this number increases with $\alpha$. Just beyond an instability the eigenmode from our linear analysis is found to be an excellent predictor of the emergent network state. Numerical simulations were performed using a 2nd-order Runge-Kutta method with linear interpolation to increase the accuracy of spike time estimates, as described in [25]. The synchronous state in a corresponding network with a smooth (fast) synapse would always be unstable, as indicated by the MSF shown in the inset of Figure 5.

For the case that $\sigma<0$ the smooth system has a stable synchronous state. In contrast the nonsmooth system is always unstable. However, the basin of attraction for the smooth case becomes vanishingly small as $\beta \rightarrow \infty$ and direct numerical simulations of the two systems (with conflicting stability predictions) would both show non-synchronous behaviour.

\section{Discussion}

Network science is a rapidly growing field of research, and plays a key role in understanding complex phenomena. In an age where large data sets are becoming more available, for example from connectomes and social media networks, it is more important than ever that we develop the tools to analyse dynamical systems on graphs. In this paper, we have made a small step in this direction with a presentation of tools for analysing the synchronous state for both smooth and nonsmooth input currents in IF networks with arbitrary topology subject to a row sum constraint. One important message that we reaffirm is that one must be careful when using a smoothing 
approach to understand the behaviour of a nonsmooth system [26]. We have seen here that a second order synapse with a fast rise time does not accurately capture the behaviour of a truly first order synapse (with a jump) when considering the linear stability of the network synchronous state. Although the spectrum from the smoothed model can approximate a subset of the spectrum from the nonsmooth problem, the latter can have further eigenvalues.

The techniques that we have presented are readily extended to cover oscillator networks without balance where non-synchronous phase-locked states are more generic. Moreover, they are also extendable to nonlinear IF models that are piece-wise linear (PWL). A very recent treatment of cluster states in PWL planar IF models with smooth synapses can be found in [27]. Another interesting extension would be to consider delays, which are especially important in large scale brain modelling [28]. The MSF has recently been extended to cover heterogenous delays [29] for smooth dynamical systems, and it would be interesting to revisit this for IF networks.

This work was supported by the Engineering and Physical Sciences Research Council [grant number EP/P007031/1]. We would like to thank Mustafa Şayli for a careful reading of this manuscript.

\section{Author contribution statement}

Y.M.L., R.T., and S.C. contributed to the design and implementation of the research, to the analysis of the results, and to the writing of the manuscript.

Open Access This is an open access article distributed under the terms of the Creative Commons Attribution License (http://creativecommons.org/licenses/by/4.0), which permits unrestricted use, distribution, and reproduction in any medium, provided the original work is properly cited.

\section{References}

1. B.W. Knight, J. Gen. Physiol. 59, 734 (1972)

2. T. Gröbler, G. Barna, P. Érdi, Biol. Cybernet. 79, 301 (1998)

3. E.M. Izhikevich, IEEE Trans. Neural. Netw. 14, 1569 (2003)

4. S. Coombes, R. Thul, K.C.A. Wedgwood, Physica D 241, 2042 (2012)

5. A.V.M Herz, J.J. Hopfield, Phys. Rev. Lett. 75, 1222 (1995)

6. P. Piedrahita, J. Borge-Holthoefer, Y. Moreno, A. Arenas, Europhys. Lett. 104, 48004 (2013)

7. P.C. Müller, Chaos Solitons Fractals 5, 1671 (1995)

8. P. Ashwin, M. Timme, Nonlinearity 18, 2035 (2005)

9. A. Corral, C.J. Pérez, A. Díaz-Guilera, A. Arenas, Phys. Rev. Lett. 75, 3697 (1995)

10. U. Ernst, K. Pawelzik, T. Geisel, Phys. Rev. Lett. 74, 1570 (1995)

11. P.H.E. Tiesinga, T.J. Sejnowski, Network Comp. Neural Syst. 12, 215 (2001)

12. P. Goel, B. Ermentrout, Physica D 163, 191 (2002)

13. M. Timme, F. Wolf, T. Geisel, Phys. Rev. Lett. 89, 258701 (2002)

14. R.E. Mirollo, S.H. Strogatz, SIAM J. Appl. Math. 50, 1645 (1990)

15. N. Brunel, M.C.W. van Rossum, Biol. Cybernet. 97, 337 (2007)

16. C. van Vreeswijk, H. Sompolinsky, Science 274, 1724 (1996)

17. P.C. Bressloff, S. Coombes, Neural Comput. 12, 91 (2000)

18. R. Leine, H. Nijmeijer, in Dynamics and Bifurcations of Non-smooth Mechanical Systems, Lecture notes in applied and computational mechanics book series (SpringerVerlag, Berlin, 2004), Vol. 18 
19. S. Coombes, Phys. Lett. A 255, 49 (1999)

20. S. Coombes, AIP Conf. Proc. 502, 88 (2000)

21. L.M. Pecora, T.L. Carroll, Phys. Rev. Lett. 80, 2109 (1998)

22. J. Ladenbauer, J. Lehnert, H. Rankoohi, T. Dahms, E. Schöll, K. Obermayer, Phys. Rev. E 88, 042713 (2013)

23. H. Kielblock, C. Kirst, M. Timme, Chaos 21, 025113 (2011)

24. S. Coombes, Y.M. Lai, M. Şayli, R. Thul, Eur. J. Appl. Math. 29, 869 (2018)

25. D. Hansel, G. Mato, C. Meunier, L. Neltner, Neural Comput. 10, 467 (1998)

26. M.R. Jeffrey, SIAM Rev. 60, 116 (2018)

27. R. Nicks, L. Chambon, S. Coombes, Phys. Rev. E 97, 032213 (2018)

28. R.G. Abeysuriya, J. Hadida, S.N. Sotiropoulos, S. Jbabdi, R. Becker, B.A.E. Hunt, M.J. Brookes, M.W. Woolrich, PLOS Comput. Biol. 14, 1 (2018)

29. A. Otto, G. Radons, D. Bachrathy, G. Orosz, Phys. Rev. E 97, 012311 (2018) 